\title{
Significance of psoas muscle thickness as an indicator of muscle atrophy in patients with hepatocellular carcinoma treated with sorafenib
}

\author{
MIO YAMASHIMA, HISAMITSU MIYAAKI, TAKUYA HONDA, \\ HIDETAKA SHIBATA, SATOSHI MIUMA, NAOTA TAURA and KAZUHIKO NAKAO \\ Department of Gastroenterology and Hepatology, Nagasaki University \\ Graduate School of Biomedical Sciences, Nagasaki 852-8501, Japan
}

Received January 28, 2017; Accepted May 3, 2017

DOI: $10.3892 / \mathrm{mco} .2017 .1321$

\begin{abstract}
Sarcopenia has been reported to be associated with the prognosis of patients with liver cirrhosis and hepatocellular carcinoma (HCC). The aim of the present study was to determine whether sarcopenia was associated with the prognosis of patients with HCC treated with sorafenib. A total of 40 patients with $\mathrm{HCC}$ who were treated with sorafenib were evaluated. As an indicator of skeletal muscle mass, transverse psoas muscle thickness (TPMT) was measured on computed tomography images at the level of the umbilicus prior to treatment initiation and after 1-3 months of treatment. Pre-TPMT/height was not associated with progression-free survival (PFS) or overall survival (OS). The change in TPMT/height prior to and following treatment was also not associated with PFS; however, the change of TPMT/height was an independent factor affecting OS $(\mathrm{P}=0.020)$. A total of 40 patients were divided into two groups depending on the degree of TPMT/height loss (mild and severe muscle atrophy groups). Patients with mild muscle atrophy exhibited a significantly longer OS compared with patients with severe muscle atrophy $(\mathrm{P}=0.045)$. Thus, the change in skeletal muscle thickness calculated as TPMT/height may be a simple predictor of survival for patients with HCC treated with sorafenib.
\end{abstract}

Correspondence to: Dr Hisamitsu Miyaaki, Department of Gastroenterology and Hepatology, Nagasaki University Graduate School of Biomedical Sciences, 1-7-1 Sakamoto, Nagasaki 852-8501, Japan

E-mail: miyaaki-hi@umin.ac.jp

Abbreviations: Vp, portal vein invasion; ALT, alanine aminotransferase; AFP, $\alpha$-fetoprotein; AFP-L3, Lens culinaris agglutinin-reactive fraction of $\alpha$-fetoprotein; DCP, des- $\gamma$-carboxy prothrombin; TPMT, transverse psoas muscle thickness; PS, performance status; CP class, Child-Pugh class; CT, computed tomography; PFS, progression-free survival; OS, overall survival

Key words: hepatocellular carcinoma, muscle atrophy, sorafenib

\section{Introduction}

Sarcopenia is defined as the loss of skeletal muscle mass and muscle dysfunction, and is caused not only by aging, but also by chronic diseases, including liver disease (1-3). The Japan Society of Hepatology set the sarcopenia assessment criteria in liver disease (4). These criteria include measuring grip strength and muscle mass by computed tomography (CT) or bioelectrical impairment analysis (BIA). Recently, an increasing number of studies have demonstrated that sarcopenia may affect the clinical state of patients with liver disease (1-3). Moreover, skeletal muscle wasting assessed by $\mathrm{CT}$ has been reported to be a prognostic marker for patients with hepatocellular carcinoma (HCC) treated with sorafenib $(5,6)$. In those reports, skeletal muscle mass was usually measured by imaging modalities, such as CT and magnetic resonance imaging. However, costly specialized software is required to measure skeletal muscle mass. Durand et al recently reported that psoas muscle thickness on CT was easily calculated as muscle mass, and this may be a predictor of mortality in patients with cirrhosis, independent of the Model for End-Stage Liver Disease (MELD) score (7). This simple method did not require specialized software.

The aim of the present study was to determine whether psoas muscle thickness predicts the prognosis of patients with HCC treated with sorafenib.

\section{Patients and methods}

Patients. This investigation was conducted in accordance with the tenets of the Declaration of Helsinki and was approved by the Ethics Committee of Nagasaki University Hospital (approval no. 16062720).

In total, $40 \mathrm{HCC}$ patients (37 men and 3 women; median age, 71.5 years) who were treated with sorafenib at Nagasaki University Hospital between May 2009 and September 2013, were enrolled in this study. The 40 patients were divided into two groups on the basis of change in TPMT/height as follows: Mild atrophy group $(n=20)$ and severe atrophy group $(n=20)$.

The diagnosis of hepatitis and liver cirrhosis was made on the basis of CT imaging and blood analysis (platelet count, hyaluronic acid and type IV collagen). Liver function was 
evaluated using the Child-Pugh (CP) classification. HCC stage was classified in accordance with the guidelines of the Liver Cancer Study Group of Japan, 5th edition (8).

Sorafenib was administered at doses of $800 \mathrm{mg}$ or $400 \mathrm{mg} / \mathrm{day}$, according to age, performance status (PS), and $\mathrm{CP}$ classification of the patients.

Measurement of skeletal muscle mass. As an indicator of skeletal muscle mass, TPMT was measured on CT at the level of the umbilicus prior to treatment initiation and after 1-3 months of treatment. Psoas mass thickness was normalized by division by height (7).

Statistical analysis. The Kaplan-Meier method was used for progression-free survival (PFS) and overall survival (OS), and Cox hazard analysis was used to determine the risk factors for PFS and OS. In addition, differences in the curves were evaluated using the long-rank test. P-values of $<0.05$ were considered to indicate statistically significant differences. Statistical analyses were conducted using StatFlex software, version 6 (Artech Co., Ltd., Osaka, Japan).

\section{Results}

Patient characteristics. The baseline characteristics and laboratory data of the patients are summarized in Table I. The liver function was normal in 2 patients, 11 patients had chronic hepatitis, and 27 had cirrhosis.

The etiologies of HCC were hepatitis B virus infection $(n=11)$, hepatitis $C$ virus infection $(n=13)$ and others $(n=16)$.

HCC stage II was diagnosed in 7 , stage III in 10, stage IVa in 10 and stage IVb in 13 patients; 4 patients exhibited portal vein invasion $(\mathrm{Vp})$

The response of HCC to treatment was evaluated by a CT scan at 1-3 months after sorafenib treatment initiation. The median TPMT/height was $18.27 \pm 3.09 \mathrm{~mm} / \mathrm{m}$ prior to sorafenib treatment. The change in TPMT/height prior to and after treatment at $1-3$ months was $0.59 \pm 0.51 \mathrm{~mm} / \mathrm{m}$.

Complete response was reported in $0 \%$, partial response in $7.5 \%(n=3)$, stable disease in $40 \%(n=16)$, and progressive disease in $42.5 \%(n=17)$ of the patients. The disease control rate according to the Response Evaluation Criteria In Solid Tumors v.1.1 (https://ctep.cancer.gov/protocoldevelopment/docs/recist_guideline.pdf) was $47.5 \%$. The median PFS was 104 days, and the median OS was 365 days.

Univariate and multivariate analysis of factors possibly associated with PFS and OS. Univariate analysis revealed that the factors associated with PFS were Vp2, $\alpha$-fetoprotein (AFP), Lens culinaris agglutinin-reactive fraction of AFP (AFP-L3), and des- $\gamma$-carboxy prothrombim (DCP). Multivariate analysis revealed that AFP-L3 was an independent factor for PFS, whereas pre-TPMT/height was not associated with PFS.

Univariate analysis revealed that factors associated with OS were PS, CP class, serum aspartate transaminase (ALT) level, serum albumin level, AFP and AFP-L3. Multivariate analysis revealed that PS and AFP-L3 were independent factors for OS. Pre-TPMT/height was not associated with OS.
Table I. Patient characteristics.

\begin{tabular}{|c|c|c|c|}
\hline Characteristics & $\begin{array}{c}N \\
(n=40)\end{array}$ & IQR & $\%$ \\
\hline Age (years) & 71.5 & 6.8 & \\
\hline \multicolumn{4}{|l|}{ Sex } \\
\hline Male & 37 & & 92.5 \\
\hline Female & 3 & & 7.5 \\
\hline \multicolumn{4}{|l|}{ Background } \\
\hline Normal liver & 2 & & 5.0 \\
\hline Chronic hepatitis & 11 & & 22.5 \\
\hline Cirrhosis & 27 & & 72.5 \\
\hline \multicolumn{4}{|l|}{ Etiology } \\
\hline HBV & 11 & & 27.5 \\
\hline $\mathrm{HCV}$ & 13 & & 32.5 \\
\hline Other & 16 & & 40.0 \\
\hline \multicolumn{4}{|l|}{ Child-Pugh score } \\
\hline 5 & 24 & & 60.0 \\
\hline 6 & 8 & & 20.0 \\
\hline 7 & 6 & & 15.0 \\
\hline 8 & 1 & & 2.5 \\
\hline 9 & 1 & & 2.5 \\
\hline \multicolumn{4}{|l|}{$\mathrm{Vp}$} \\
\hline $\mathrm{Vp}^{-}$ & 36 & & 90.0 \\
\hline $\mathrm{Vp}^{+}$ & 4 & & 10.0 \\
\hline \multicolumn{4}{|l|}{ HCC stage } \\
\hline II & 7 & & 17.5 \\
\hline III & 10 & & 25.0 \\
\hline IVa & 10 & & 25.0 \\
\hline $\mathrm{IVb}$ & 13 & & 32.5 \\
\hline ALT (IU/1) & 33.5 & 21.8 & \\
\hline T-Bil (mg/dl) & 0.8 & 0.26 & \\
\hline $\mathrm{Alb}(\mathrm{g} / \mathrm{dl})$ & 3.6 & 0.28 & \\
\hline $\operatorname{PLT}\left(\times 10^{4} / \mu 1\right)$ & 13.2 & 3.45 & \\
\hline $\operatorname{AFP}(\mathrm{ng} / \mathrm{ml})$ & 26 & 341.7 & \\
\hline AFP-L3 (\%) & 12.8 & 15.7 & \\
\hline $\mathrm{DCP}(\mathrm{mAU} / \mathrm{ml})$ & 241 & 3226.5 & \\
\hline Height (m) & 1.628 & 0.054 & \\
\hline Pre-TPMT/height (mm/m) & 18.27 & 3.09 & \\
\hline Change in TPMT/height $(\mathrm{mm} / \mathrm{m})$ & 0.59 & 0.51 & \\
\hline
\end{tabular}

Values are presented as median (IQR). IQR, interquartile range; $\mathrm{HBV}$, hepatitis B virus; $\mathrm{HCV}$, hepatitis C virus; $\mathrm{HCC}$, hepatocellular carcinoma; Vp, portal vein invasion; ALT, alanine aminotransferase; T-Bil, total bilirubin; Alb, albumin; PLT, platelet count; AFP, $\alpha$-fetoprotein; AFP-L3, Lens culinaris agglutinin-reactive fraction of AFP; DCP, des- $\gamma$-carboxy prothrombin; TPMT, transverse psoas muscle thickness.

Furthermore, the association between each factor and the change in TPMT/height before and after treatment was examined. Univariate analysis revealed that the factors associated with PFS were Vp2, AFP, AFP-L3 and DCP. Multivariate analysis revealed that PS and AFP-L3 were independent factors 
Table II. Univariate and multivariate analysis of possible risk factors for progression-free survival.

\begin{tabular}{|c|c|c|c|c|}
\hline \multirow[b]{2}{*}{ Variables $(n=40)$} & \multicolumn{2}{|c|}{ Univariate } & \multicolumn{2}{|c|}{ Multivariate } \\
\hline & P-value & HR & P-value & HR \\
\hline Age (years) & 0.14267 & & & \\
\hline Gender (male vs. female) & 0.33959 & & & \\
\hline$P S \geq(0$ vs.$\geq 1)$ & 0.40901 & & & \\
\hline CP score & 0.09731 & & & \\
\hline HCC stage (II, III vs. IVa, IVb) & 0.13077 & & & \\
\hline Vp2 (+ vs. - ) & 0.03243 & 5.62262 & 0.34786 & 2.75054 \\
\hline ALT (IU/ml) & 0.11228 & & & \\
\hline $\operatorname{PLT}\left(\times 10^{4} / \mu 1\right)$ & 0.39513 & & & \\
\hline $\mathrm{Alb}(\mathrm{g} / \mathrm{dl})$ & 0.27394 & & & \\
\hline $\operatorname{AFP}(n g / m l)$ & 0.03676 & 1.00001 & 0.7604 & 1.00000 \\
\hline AFP-L3 (< vs. $\geq 10 \%)$ & 0.00940 & 3.04096 & 0.04295 & 2.49557 \\
\hline $\mathrm{DCP}(\mathrm{mAU} / \mathrm{ml})$ & 0.02016 & 1.00006 & 0.29361 & 1.00004 \\
\hline Pre-TPMT/height (mm/m) & 0.50048 & & & \\
\hline Change in TPMT/height (mm/m) & 0.48890 & & & \\
\hline
\end{tabular}

HCC, hepatocellular carcinoma; HR, hazard ratio; PS, performance status; CP score, Child-Pugh score; Vp, portal vein invasion; ALT, alanine aminotransferase; PLT, platelet count; Alb, albumin; AFP, $\alpha$-fetoprotein; AFP-L3, Lens culinaris agglutinin-reactive fraction of AFP; DCP, des- $\gamma$-carboxy prothrombim; TPMT, transverse psoas muscle thickness.

Table III. Univariate and multivariate analysis of possible risk factors for overall survival.

\begin{tabular}{|c|c|c|c|c|}
\hline \multirow[b]{2}{*}{ Variables $(n=40)$} & \multicolumn{2}{|c|}{ Univariate } & \multicolumn{2}{|c|}{ Multivariate } \\
\hline & P-value & HR & P-value & HR \\
\hline Age (years) & 0.42107 & & & \\
\hline Gender (male vs. female) & 0.58509 & & & \\
\hline PS $(0$ vs. $\geq 1)$ & 0.01525 & 2.48488 & 0.03273 & 2.79015 \\
\hline CP score & 0.03651 & 1.58368 & 0.29636 & \\
\hline HCC stage (II, III vs. IVa, IVb) & 0.47593 & & & \\
\hline Vp2 (+ vs. -) & 0.50677 & & & \\
\hline Alt (IU/ml) & 0.00074 & 1.01986 & 0.30556 & \\
\hline $\operatorname{PLT}\left(\times 10^{4} / \mu 1\right)$ & 0.06438 & & & \\
\hline $\mathrm{Alb}(\mathrm{g} / \mathrm{dl})$ & 0.03263 & 0.38263 & 0.90507 & \\
\hline $\operatorname{AFP}(n g / m l)$ & 0.00697 & 1.00002 & 0.28770 & \\
\hline AFP-L3 (<vs. $\geq 10 \%)$ & 0.02407 & 2.39921 & 0.12226 & \\
\hline $\mathrm{DCP}(\mathrm{mAU} / \mathrm{ml})$ & 0.65623 & & & \\
\hline Pre-TPMT/height (mm/m) & 0.54100 & & & \\
\hline Change in TPMT/height $(\mathrm{mm} / \mathrm{m})$ & 0.00100 & 1.95459 & 0.02017 & 2.27120 \\
\hline
\end{tabular}

for PFS. The change in TPMT/height was not associated with PFS (Table II). Univariate analysis revealed that the factors associated with OS were PS, CP class, serum ALT level, serum albumin level, AFP, AFP-L3 and the change in TPMT/height. Multivariate analysis revealed that PS and the change of TPMT/height were independent factor for OS (Table III).
Moreover, the association was examined when the patients were limited to those with $\mathrm{CP}$ score A $(n=32)$. The change in TPMT/height was also an independent factor for OS $(\mathrm{P}=0.0439$, hazard ratio $=1.99271$, data not shown).

The cut-off value for the difference in TPMT/height was $0.59 \mathrm{~mm} / \mathrm{m}$. Patients in the mild atrophy group had a 

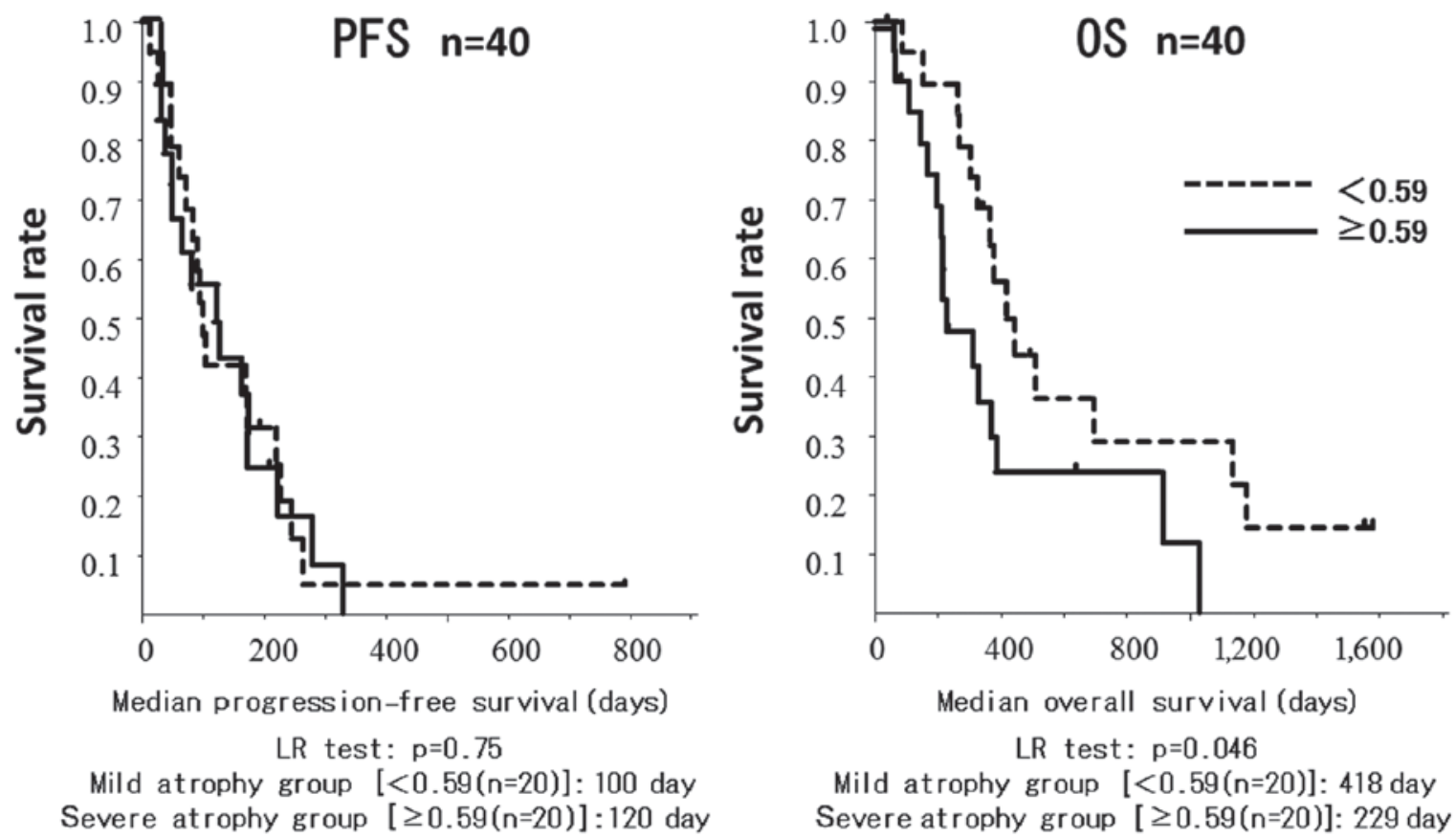

Figure 1. Kaplan-Meier curves for progression-free survival (PFS) and overall survival (OS) of the mild and severe atrophy groups. Patients in the mild atrophy group had a significantly longer OS compared with patients in the severe atrophy group $(\mathrm{P}=0.045)$.

significantly longer OS compared with patients in the severe atrophy group $(418 \pm 0.12$ vs. $229 \pm 0.11$ days, respectively; $\mathrm{P}=0.045$; Fig. 1).

\section{Discussion}

A number of previous studies reported the efficacy of sorafenib for the treatment of advanced HCC (9-11). However, sorafenib is costly and has been associated with severe adverse effects. Therefore, it is important to establish predictive factors for sorafenib therapy. Previous studies demonstrated that several factors, such as Eastern Cooperative Oncology Group PS and $\mathrm{CP}$ class A status were predictive factors for patients with $\mathrm{HCC}$ receiving sorafenib therapy $(11,12)$. In recent reports, skeletal muscle mass loss was found to be significantly associated with malnutrition and poor liver function $(13,14)$. Our study indicated that skeletal muscle thickness prior to treatment was not associated with PFS or OS in patients treated with sorafenib. However, the decreased skeletal muscle thickness prior to treatment was found to be associated with OS. Multivariate analysis also demonstrated that decreased skeletal muscle thickness and PS were independent predictors for survival time. This result indicated that these malnutritional factors were important for the survival of patients with HCC treated with sorafenib.

Several recent reports indicated that decreased skeletal muscle mass was an important prognostic factor in patients with chronic liver disease and HCC $(2,3,5,6)$. These results suggested that preventing reductions in skeletal muscle thickness may improve the OS of patients treated with sorafenib. The usefulness of nutritional support, such as branched-chain amino acids (BCAA), and exercise therapies to prevent the decrease in skeletal muscle mass were reported $(3,15,16)$. Only in patients with cirrhosis, supplementation with BCAA prevented skeletal muscle loss (BCAA vs. without BCAA, $0.64 \pm 1.04$ vs. $1.56 \pm 1.77$, respectively; $\mathrm{P}=0.10$; data not shown).

Dual energy X-ray absorptiometry (DXA) (17), BIA (18) and CT $(19,20)$ have been widely used to measure skeletal muscle mass. However, since DXA and BIA are sensitive to water composition, they are not considered suitable for patients with cirrhosis. Moreover, most institutions do not have the necessary equipment to perform these examinations. CT scans are commonly used to assess the status of HCC patients treated with sorafenib. Therefore, a number of used CT to assess patients with chronic liver disease or HCC.

The psoas area at the L3 vertebral level was used in studies on sarcopenia, as it is considered to be well-correlated with and may substitute whole-body muscle mass. However, the measurement of skeletal muscle mass in the psoas area requires specialized software. Durand $e t$ al reported that axial and transverse psoas thickness on CT scan at the level of the umbilicus was predictive of mortality in patients with liver cirrhosis, independent of the MELD score (7). This method did not require specific software and could be calculated over a short time on real time and on an outpatient basis; therefore, it was selected as an indicator of skeletal muscle mass.

The present study had several limitations, including the single-centered design and its small sample size. To validate the findings, large multicenter studies with a larger sample size are required. In addition, as TPMT/height was measured manually, the accuracy of this method may have been affected by different examiners.

In conclusion, the results of the present study suggest that the change in skeletal muscle thickness, which was calculated as TPMT/height, may be a predictor of survival in HCC patients treated with sorafenib, and preventing skeletal muscle loss may improve the outcome of such patients. 


\section{References}

1. Tandon P, Ney M, Irwin I, Ma MM, Gramlich L, Bain VG, Esfandiari N, Baracos V, Montano-Loza AJ and Myers RP: Severe muscle depletion in patients on the liver transplant wait list: Its prevalence and independent prognostic value. Liver Transpl 18: 1209-1216, 2012.

2. Montano-Loza AJ, Meza-Junco J, Prado CM, Lieffers JR, Baracos VE, Bain VG and Sawyer MB: Muscle wasting is associated with mortality in patients with cirrhosis. Clin Gastroenterol Hepatol 10: 166-173.e1, 2012.

3. Hanai T, Shiraki M, Nishimura K, Ohnishi S, Imai K, Suetsugu A, Takai K, Shimizu M and Moriwaki H: Sarcopenia impairs prognosis of patients with liver cirrhosis. Nutrition 31: 193-199, 2015

4. Nishikawa H, Shiraki M, Hiramatsu A, Moriya K, Hino K and Nishiguchi S: Japan Society of Hepatology guidelines for sarcopenia in liver disease (1st edition): Recommendation from the working group for creation of sarcopenia assessment criteria Hepatol Res 46: 951-963, 2016.

5. Imai K, Takai K, Hanai T, Ideta T, Miyazaki T, Kochi T, Suetsugu A, Shiraki M and Shimizu M: Skeletal muscle depletion predicts the prognosis of patients with hepatocellular carcinoma treated with sorafenib. Int J Mol Sci 16: 9612-9624, 2015.

6. Hiraoka A, Hirooka M, Koizumi Y, Izumoto H, Ueki H, Kaneto M, Kitahata S, Aibiki T, Tomida H, Miyamoto Y, et al: Muscle volume loss as a prognostic marker in hepatocellular carcinoma patients treated with sorafenib. Hepatol Res 47: 558-565, 2017.

7. Durand F, Buyse S, Francoz C, Laouénan C, Bruno O, Belghiti J, Moreau R, Vilgrain V and Valla D: Prognostic value of muscle atrophy in cirrhosis using psoas muscle thickness on computed tomography. J Hepatol 60: 1151-1157, 2014.

8. Liver Cancer Study Group of Japan, corp-author. General Rules for the Clinical and Pathological Study of Primary Liver Cancer. 5th edition. Kanehara \& Co., Ltd. Tokyo, p24, 2009.

9. Llovet JM, Ricci S, Mazzaferro V, Hilgard P, Gane E, Blanc JF, de Oliveira AC, Santoro A, Raoul JL, Forner A, et al: Sorafenib in advanced hepatocellular carcinoma. N Engl J Med 359: 378-390, 2008

10. Cheng AL, Kang YK, Chen Z, Tsao CJ, Qin S, Kim JS, Luo R, Feng J, Ye S, Yang TS, et al: Efficacy and safety of sorafenib in patients in the Asia-Pacific region with advanced hepatocellular carcinoma: A phase III randomised, double-blind, placebo-controlled trial. Lancet Oncol 10: 25-34, 2009.
11. Bruix J, Raoul JL, Sherman M, Mazzaferro V, Bolondi L, Craxi A, Galle PR, Santoro A, Beaugrand M, Sangiovanni A, et al: Efficacy and safety of sorafenib in patients with advanced hepatocellular carcinoma: Subanalyses of a phase III trial. J Hepatol 57: 821-829, 2012.

12. Hollebecque A, Cattan S, Romano O, Sergent G, Mourad A, Louvet A, Dharancy S, Boleslawski E, Truant S, Pruvot FR, et al: Safety and efficacy of sorafenib in hepatocellular carcinoma: The impact of the Child-Pugh score. Aliment Pharmacol Ther 34: 1193-1201, 2011.

13. Montano-Loza AJ: Clinical relevance of sarcopenia in patients with cirrhosis. World J Gastroenterol 20: 8061-8071, 2014.

14. Periyalwar P and Dasarathy S: Malnutrition in cirrhosis: Contribution and consequences of sarcopenia on metabolic and clinical responses. Clin Liver Dis 16: 95-131, 2012.

15. Toshikuni N, Arisawa T and Tsutsumi M: Nutrition and exercise in the management of liver cirrhosis. World J Gastroenterol 20: 7286-7297, 2014

16. Kaibori M, Ishizaki M, Matsui K, Nakatake R, Yoshiuchi S, Kimura Y and Kwon AH: Perioperative exercise for chronic liver injury patients with hepatocellular carcinoma undergoing hepatectomy. Am J Surg 206: 202-209, 2013.

17. Sanada K, Miyachi M, Tanimoto M, Yamamoto K, Murakami H, Okumura S, Gando Y, Suzuki K, Tabata I and Higuchi M: A cross-sectional study of sarcopenia in Japanese men and women: Reference values and association with cardiovascular risk factors. Eur J Appl Physiol 110: 57-65, 2010.

18. Tanimoto Y, Watanabe M, Sun W, Hirota C, Sugiura Y, Kono R, Saito $\mathrm{M}$ and Kono K: Association between muscle mass and disability in performing instrumental activities of daily living (IADL) in community-dwelling elderly in Japan. Arch Gerontol Geriatr 54: e230-e233, 2012.

19. Hiraoka A, Aibiki T, Okudaira T, Toshimori A, Kawamura T, Nakahara H, Suga Y, Azemoto N, Miyata H, Miyamoto Y, et al: Muscle atrophy as pre-sarcopenia in Japanese patients with chronic liver disease: Computed tomography is useful for evaluation. J Gastroenterol 50: 1206-1213, 2015.

20. Yoshizumi T, Shirabe K, Nakagawara H, Ikegami T, Harimoto N, Toshima T, Yamashita Y, Ikeda T, Soejima Y and Maehara Y: Skeletal muscle area correlates with body surface area in healthy adults. Hepatol Res 44: 313-318, 2014. 